\title{
Fault Tolerant Deployment and Topology Control in Wireless Networks
}

\author{
Xiang-Yang Li Peng-Jun Wan Yu Wang Chih-Wei Yi \\ Dept. of Computer Science, Illinois Institute of Technology \\ 10 W. 31st Street, Chicago, IL 60616 \\ \{xli,wan\}@cs.iit.edu, \{wangyu1,yichihw\}@iit.edu
}

\begin{abstract}
This paper investigate fault tolerance for wireless ad hoc networks. We consider a large-scale of wireless networks whose nodes are distributed randomly in a unit-area square region. Given $n$ wireless nodes $V$, each with transmission range $r_{n}$, the wireless networks are often modeled by graph $G\left(V, r_{n}\right)$ in which two nodes are connected if their Euclidean distance is no more than $r_{n}$.

We first consider how the transmission range is related with the number of nodes in a fixed area such that the resulted network can sustain $k$ fault nodes with high probability. We show that, for a unit-area square region, the probability that the network $G\left(V, r_{n}\right)$ is $(k+1)$-connected is at least $e^{-e^{-\alpha}}$ when the transmission radius $r_{n}$ satisfies $n \pi r_{n}^{2} \geq \ln n+(2 k-1) \ln \ln n-2 \ln k !+2 \alpha$ for $k>0$ and $n$ sufficiently large. This result also applies to mobile networks when the moving of wireless nodes always generates randomly distributed positions. Our simulations show that $n$ should be larger than 500 if $k=2$ or 3 and $\alpha=\log n$; and $n$ should be larger than 2500 if $k=2$ or 3 and $\alpha=\log \log n$.

We then present a localized method to control the network topology given a $(k+1)$-faults tolerant deployment $G\left(V, r_{n}\right)$ of wireless nodes such that the resulting topology is still $(k+1)$-faults tolerant but with $O(k n)$ communication links maintained. We show that the constructed topology is also a length spanner. Here a subgraph $H$ is spanner of graph $G$, if for any two nodes, the length of the shortest path connecting them in $H$ is no more than a small constant factor of the length of the shortest path connecting them in $G$.

Finally, we conduct some simulations to study the practical transmission range to achieve certain probability of $k$-connected when $n$ is not large enough.
\end{abstract}

\section{Categories and Subject Descriptors}

C.2.1 [Network Architecture and Design]: Wireless communication; G.2.2 [Graph Theory]: Network problems; G.3 [Probability and Statistics]

Permission to make digital or hard copies of all or part of this work for personal or classroom use is granted without fee provided that copies are not made or distributed for profit or commercial advantage and that copies bear this notice and the full citation on the first page. To copy otherwise, to republish, to post on servers or to redistribute to lists, requires prior specific permission and/or a fee.

MobiHoc'03, June 1-3, 2003, Annapolis, Maryland, USA.

Copyright 2003 ACM 1-58113-684-6/03/0006 ...\$5.00.

\section{General Terms}

Algorithms, Design, Theory

\section{Keywords}

Fault tolerance, connectivity, topology control, wireless ad hoc networks

\section{INTRODUCTION}

Wireless ad hoc networks are also called packet radio networks in the early 70's. While many fundamental ideas existed about twenty to thirty years ago, recent years we see tremendous research activity in wireless ad hoc networks due to its applications in various situations such as battlefield, emergency relief, and so on. Mobile wireless networking enjoys a great advantage over the wired networking counterpart because it can be formed in a spontaneous way for various applications.

There are no wired infrastructures or cellular networks in wireless ad hoc network. Each mobile node ${ }^{1}$ has an adjustable transmission range. Node $v$ can receive the signal from node $u$ if node $v$ is within the transmission range of the sender $u$. Otherwise, two nodes communicate through multi-hop ad hoc wireless links by using intermediate nodes to relay the message. Consequently, each node in the wireless network also acts as a router, forwarding data packets for other nodes. We consider that each wireless node has an omni-directional antenna. This is attractive for a single transmission of a node can be received by many nodes within its vicinity which, we assume, is a disk centered at the node.

Hundreds of protocols $[5,6,12,17,24,27,28,31]$ that take the unique characteristics of wireless ad hoc networks have been developed. Among them energy efficiency, routing and MAC layer protocols have attracted most attention. One of the remaining fundamental and critical issues is to have fault-tolerant network deployment without sacrificing the spectrum reusing property. In other words, the network should support multiple disjoint paths connecting every pair of nodes. Obviously, we can increase the transmission range of all nodes to increase the fault-tolerance of the network. However, increasing the transmission range will increase the power consumption of every node. As power is a scarce resource in wireless networks, it is important to save the power consumption without losing the network connectivity. The universal minimum power used by all wireless nodes such that the induced network topology is connected is called the critical power.

Determining the critical power was studied by several researchers $[11,18,26,29]$ recently when the wireless nodes are statically dis-

\footnotetext{
${ }^{1}$ In this paper the term node often represents a network device, vertex is a graph term, and point is a geometry term. We often interchange them if no confusion is caused.
} 
tributed. Both [26] and [29] use the power assignment induced by the longest incident edge of the Euclidean minimum spanning tree over wireless nodes $V$. It was proved by Penrose [20] that, given a set of points uniformly and randomly distributed in a unit-area square, the longest edge of the minimum spanning tree asymptotically equals to the longest edge of the nearest neighbor graph. Since the nearest neighbor can be found locally, we can determine the critical power asymptotically using a localized method instead of constructing the minimum spanning tree if the wireless devices are randomly and uniformly distributed in a unit area square. Notice that, to form the nearest neighbor graph, every node $u$ selects the nearest neighbor inside its maximal transmission range, and tells its neighbors about the selection. This can be done locally. Recently, Narayanaswamy et al. [18] proposed and implemented the first power control protocol (COMPOW) which guarantees connectivity of wireless ad hoc networks and has been tested on a real wireless test-bed.

Although determining the critical power for static wireless ad hoc networks is well-studied, it remains to study the critical power for connectivity for mobile wireless networks. As the wireless nodes move around, it is impossible to have a unanimous critical power to guarantee the connectivity for all instances of the network configuration. Thus, we need to find a critical power, if possible, at which each node has to transmit to guarantee the connectivity of the network almost surely, i.e., with high probability almost 1 . For simplicity, we assume that the wireless devices are distributed in a unit area square (or disk) according to some distribution function, e.g., uniform distribution or Poisson process. Additionally, assuming that the movement of wireless devices still keeps them the same distribution (uniform or Poisson process). Gupta and $\mathrm{Ku}$ mar [11] showed that there is a critical power almost surely when the wireless nodes are randomly and uniformly distributed in a unit area disk. The result by Penrose [20] implies the same conclusion. Moreover, Penrose [20] gave the probability of the network to be connected if the transmission radius is set as a positive real number $r$ and $n$ goes to infinity.

Let $G(V, r)$ be the graph defined on $V$ with edges $u v \in E$ iff $\|u v\| \leq r$. Here $\|u v\|$ is the Euclidean distance between nodes $u$ and $v$. Let $\mathcal{G}_{\Omega}\left(\mathcal{X}_{n}, r_{n}\right)$ be the set of graphs $G\left(V, r_{n}\right)$ for $n$ nodes $V$ that are uniformly and independently distributed in a twodimensional region $\Omega$, which could be a unit-area disk $\mathcal{D}$ or a unit square $\mathcal{C}$ with center at the origin. The problem considered by Gupta and Kumar [11] is then to determine the value of $r_{n}$ such that a random graph in $\mathcal{G}_{\mathcal{D}}\left(\mathcal{X}_{n}, r_{n}\right)$ is asymptotically connected with probability one as $n$ goes infinity. Let $P_{\Omega, k}\left(\mathcal{X}_{n}, r_{n}\right)$ be the probability that a graph in $\mathcal{G}_{\Omega}\left(\mathcal{X}_{n}, r_{n}\right)$ is $k$-connected. Then Gupta and Kumar [11] showed that if $n \pi \cdot r_{n}^{2}=\ln n+c(n)$, then $P_{\Omega, 1}\left(n, r_{n}\right) \rightarrow 1$ iff $c(n) \rightarrow+\infty$ as $n$ goes infinity. The result by Penrose [20] implies a stronger result: if $n \pi \cdot r_{n}^{2}=\ln n+\alpha$, then $P_{1}\left(n, r_{n}\right)=e^{-e^{-\alpha}}$ as $n$ goes infinity.

Fault tolerance is one of the central challenges in designing the wireless ad hoc networks. To make fault tolerance possible, first of all, the underlying network topology must have multiple disjoint paths to connect any two given wireless devices. Here the path could be vertex disjoint or edge disjoint. We use the vertex disjoint multiple paths in this paper considering the communication nature of the wireless networks. In this paper, we are interested in what is the condition of $r_{n}$ such that the underlying network topology $G\left(V, r_{n}\right)$ is $k$-connected almost surely when $V$ is uniformly and randomly distributed over a two-dimensional domain $\Omega$. For simplicity, we assume that the geometry domain $\Omega$ is a unit square $\mathcal{C}$. Gupta and Kumar [11] basically studied the connectivity problem for $k=1$ and $\Omega$ being a unit-area disk.
We show that, given $n$ points randomly distributed in a unit square $\mathcal{C}$, if the transmission range $r_{n}$ satisfies $n \pi \cdot r_{n}^{2} \geq \ln n+$ $(2 k-1) \ln \ln n-2 \ln k !+\alpha+2 \ln \frac{8 k}{2^{k} \sqrt{\pi}}$, then $G\left(V, r_{n}\right)$ is $(k+1)$ connected with probability at least $e^{-e^{-\alpha}}$ as $n$ goes infinity. Notice that, this result is analogous to the corresponding result for Bernoulli graphs $\mathcal{G}(n, p)$; See [4]. A similar result was presented by Penrose $[20,22]$ for the toroidal model instead of the Euclidean model. He showed that, the hitting radius $r_{n}$ such that the graph $G\left(V, r_{n}\right)$ is $(k+1)$-connected satisfies

$$
\lim _{n \rightarrow \infty} \operatorname{Pr}\left(n \pi r_{n}^{2} \leq \ln n+k \ln \ln n-\ln k !+\alpha\right)=e^{-e^{-\alpha}} .
$$

The toroidal metric is used to eliminate boundary effects. Recently, Bettstetter [2] also investigated the minimum node degree and $k$ connectivity and constructed various simulations to verify his analytical expressions. However his theoretical result does not consider the boundary effects, which is impossible in real networks.

Our theoretical value gives us insight on how to set the transmission radius to achieve the $k$-connectivity with certain probability for a network of $n$ devices; or how many devices are needed to achieve the $k$-connectivity with certain probability when the transmission range of each device is a fixed value. This result also applies to mobile networks when the moving of wireless nodes always generate randomly (or Poisson process) distributed node positions. Our result has applications in system design of large scale wireless networks. For example, for setting up a sensor network monitoring a certain region, we should deploy how many sensors to have a multiple connected network knowing each sensor can transmit a range $r_{0}$. Notice that our result holds only when the number of wireless devices $n$ goes to infinity, which is difficult to deploy practically. We then conduct extensive simulations to study the transmission radius achieving $k$-connectivity with certain probability for practical settings. The relation between the minimum node degree and the connectivity of graph $G(V, r)$ is also studied here.

After deciding the critical power for $k$-connectivity for mobile wireless networks, another important issue is topology control. Due to the nodes' limited resource in wireless ad hoc networks, the scalability is crucial for network operations. One effective approach is to maintain only a linear number of links using a localized construction method. However, this sparseness of the constructed network topology should not compromise on the fault tolerance and compromise too much on the power consumptions for both unicast and broadcast/multicast communications. We then present a localized method to control the network topology given a $k$-faults tolerant deployment of wireless nodes such that the resulting topology is still fault tolerant but with much fewer communication links maintained. We show that the constructed topology has only linear number of links and is a length spanner.

The remaining of the paper is organized as follows. In Section 2, we review some previous results studying the transition phenomena for wireless networks. Section 3 studies the critical transmission range for $k$-connectivity of the wireless ad hoc networks when the wireless nodes are randomly and uniformly distributed in a unitarea square $\mathcal{C}$. In Section 4, we present a localized method to control the network topology. The resulting topology can not only sustain $k$ node faults, but also approximates the original unit disk graph well in terms of the energy consumption. Our experimental results presented in 5 will verify our theoretical results. We conclude our paper and discuss possible future research directions in Section 6.

\section{PRELIMINARIES}

Before reviewing previous results, we first recall some basic definition used in this paper. Given an event $Y$, let $\operatorname{Pr}(Y)$ be the prob- 
ability of $Y$. We denote the expected value of a random variable $X$ by $E[X]$, i.e., $E[X]=\sum_{x} x \cdot \operatorname{Pr}(X=x)$ for a discrete variable. As standard, we write log for base-2 logarithm and ln for natural logarithm. We say a function $f(n) \rightarrow a$ if $\lim _{n \rightarrow \infty} f(n)=a$.

\subsection{Point Process}

In this paper we use two different point processes: the random point process and the homogeneous Poisson process of intensity.

A point process is said to be a uniform random point process, denoted by $\mathcal{X}_{n}$, in a region $\Omega$ if it consists of $n$ independent points each of which is uniformly and randomly distributed over $\Omega$.

The standard probabilistic model of homogeneous Poisson process is characterized by the property that the number of nodes in a region is a random variable depending only on the area (or volume in higher dimensions) of the region. In other words,

- The probability that there are exactly $k$ nodes appearing in any region $\Psi$ of area $A$ is $\frac{(\lambda A)^{k}}{k !} \cdot e^{-\lambda A}$.

- For any region $\Psi$, the conditional distribution of nodes in $\Psi$ given that exactly $k$ nodes in the region is joint uniform.

- The numbers of nodes in disjoint sets are independent.

Here after, we let $\mathcal{P}_{n}$ be a homogeneous Poisson process of intensity $n$ on the unit square $\mathcal{C}=[-0.5,0.5] \times[-0.5,0.5]$.

\subsection{Connectivity and Minimum Degree}

A graph is called $k$-vertex connected ( $k$-connected for simplicity) if, for each pair of vertices, there are $k$ mutually vertex disjoint paths (except end-vertices) connecting them. Equivalently, a graph is $k$-connected if there is no a set of $k-1$ nodes whose removal will partition the network into at least two components. Thus, a $k$ connected wireless network can sustain the failure of $k-1$ nodes. A graph is called $k$-edge connected if, for each pair of vertices, there are $k$ mutually edge disjoint paths connecting them. The vertex connectivity, denoted by $\kappa(G)$, of a graph $G$ is the maximum $k$ such that $G$ is $k$ vertex connected. The edge connectivity, denoted by $\xi(G)$, of a graph $G$ is the maximum $k$ such that $G$ is $k$ edge connected. The minimum degree of a graph $G$ is denoted by $\delta(G)$ and the maximum degree of a graph $G$ is denoted by $\Delta(G)$. Clearly, for any graph $G, \kappa(G) \leq \xi(G) \leq \delta(G) \leq \Delta(G)$. We will omit the symbol $G$ in the above notations if it is clear from the context.

A graph property is called monotone increasing if $G$ has such property then all graphs on the same vertex set containing $G$ as a subgraph have this property. Let $\mathcal{Q}$ be any monotone increasing property of graphs, for example, the connectivity, the $k$-edge connectivity, the $k$-vertex connectivity, the minimum node degree at least $k$, and so on. The hitting radius $\varrho(V, \mathcal{Q})$ is the infimum of all $r$ such that graph $G(V, r)$ has property $\mathcal{Q}$. For example, $\varrho(V, \kappa \geq k)$ is the minimum radius $r$ such that $G(V, r)$ is at least $k$ vertex connected; $\varrho(V, \delta \geq k)$ is the minimum radius $r$ at which the graph $G(V, r)$ has the minimum degree at least $k$. Obviously, for any $V$,

$$
\varrho(V, \kappa \geq k) \geq \varrho(V, \delta \geq k) .
$$

Penrose [22] showed that these two hitting radii are asymptotically same for $n$ points $V$ randomly and uniformly distributed in a unit square and $n$ goes infinity.

\subsection{Literature Review}

The connectivity of random graphs, especially the geometric graphs and its variations, have been considered in the random graph theory literature [4], in the stochastic geometry literature [7, 20, 21,
$22,23]$, and the wireless ad hoc network literature $[2,3,9,10,11$, $18,19,30,33]$.

Let's first consider the connectivity problem. Given $n$ nodes $V$ randomly and independently distributed in a unit-area disk $\mathcal{D}$, Gupta and Kumar [11] showed that $G\left(V, r_{n}\right)$ is connected almost surely if $n \pi \cdot r_{n}^{2} \geq \ln n+c(n)$ for any $c(n)$ with $c(n) \rightarrow \infty$ as $n$ goes infinity. Notice this bound is tight as they also proved that $\mathcal{G}\left(\mathcal{X}_{n}, r_{n}\right)$ is asymptotically disconnected with positive probability if $n \pi \cdot r_{n}^{2}=\ln n+c(n)$ and $\lim \sup _{n} c(n)<+\infty$.

Notice that, they actually derived their results for a homogeneous Poisson process of points in $\mathcal{D}$ instead of the independent and uniform point process. They showed that the difference between them is negligible. Additionally, it is easy to show that the same result holds if the geometry domain in which the wireless nodes are distributed is a unit-area square $\mathcal{C}$ instead of the unit-area disk $\mathcal{D}$.

Independently, Penrose [20] showed that the longest edge $M_{n}$ of the minimum spanning tree of $n$ points randomly and uniformly distributed in a unit area square $\mathcal{C}$ satisfies that

$$
\lim _{n \rightarrow \infty} \operatorname{Pr}\left(n \pi M_{n}^{2}-\ln n \leq \alpha\right)=e^{-e^{-\alpha}},
$$

for any real number $\alpha$. Remember that, the longest edge of EMST is always the critical power [26, 29]. Thus, the result in [20] is actually stronger than that in [11] since it will give the probability that the network is connected. For example, if we set $\alpha=\ln \ln n$, we have $\operatorname{Pr}\left(n \pi M_{n}^{2} \leq \ln n+\ln \ln n\right)=e^{-1 / \ln n}$. It implies that the network is connected with probability at least $e^{-1 / \ln n}$ if the transmission radius of each node $r_{n}$ satisfies $n \pi r_{n}^{2}=\ln n+\ln \ln n$. Notice that $e^{-1 / \ln n}>1-\frac{1}{\ln n}$ from $e^{-x}>1-x$ for $x>0$. By setting $\alpha=\ln n$, the probability that the graph $G\left(V, r_{n}\right)$ is connected is at least $e^{-1 / n}>1-\frac{1}{n}$, where $n \pi r_{n}^{2}=2 \ln n$. Notice that the above probability is only true when $n$ goes to infinity. When $n$ is a finite number, then the probability of the graph being connected is smaller. In this paper, we will present the first experimental study of the probability of the graph $G\left(V, r_{n}\right)$ being connected for finite number $n$.

We then review the results concerning the $k$-connectivity of a random graph. It was proved by Penrose [22] that, given any metric $l_{p}$ with $2 \leq p \leq \infty$ and any positive integer $k$,

$$
\lim _{n \rightarrow \infty} \operatorname{Pr}\left(\varrho\left(\mathcal{X}_{n}, \kappa \geq k\right)=\varrho\left(\mathcal{X}_{n}, \delta \geq k\right)\right)=1 .
$$

This result is analogous to the well-known results in the random graph theory [4] that graph becomes $k$ vertex connected when it achieves the minimum degree $k$ if we add the edges randomly and uniformly from $\left(\begin{array}{l}n \\ 2\end{array}\right)$ ! possibilities. This result by Penrose [22] says that a graph of $G\left(\mathcal{X}_{n}, r\right)$ becomes $k$-connected almost surely at the moment it has minimum degree $k$ by letting $r$ go from 0 to $\infty$. However, this result does not imply that, to guarantee a graph over $n$ points $k$-connected almost surely, we only have to connect every node to its $k$ nearest neighbors.

Recently, Bettstetter [2] investigated the minimum node degree and $k$-connectivity when network is modeled by geometric random graph. He also constructed various simulations to verify his analytical expressions. However, his analytical derivation assumed an infinite large area (or toroidal model) to avoid the boundary effects, which is impossible in real wireless systems.

Xue and Kumar [33] proved that, to guarantee a geometry graph over $V$ connected, the number of nearest neighbors that every node has to connect is asymptotically $\Theta(\ln n)$. We conjecture that, given $n$ random points $V$ over a unit-area square, to guarantee a geometry graph over $V(k+1)$-connected, the number of nearest neighbors that every node has to connect is asymptotically $\Theta(\ln n+(2 k-$ $1) \ln \ln n)$. We leave this as future work. Dette and Henze [7] 
studied the maximum length of the graph by connecting every node to its $k$ nearest neighbors asymptotically, for constant $k$.

Similarly, instead of considering $\mathcal{X}_{n}$, Penrose also considered a homogeneous Poisson point process with intensity $n$ on the unitarea square $\mathcal{C}$. Penrose gave loose upper and lower bound on the hitting radius $r_{n}=\varrho\left(\mathcal{P}_{n}, \delta \geq k\right)$ as $\frac{\ln n}{2^{d+1}} \leq n r_{n}^{d} \leq d ! 2 \ln n$ for homogeneous Poisson point process on a $d$-dimensional unit cube, This result is too loose. More importantly, the parameter $k$ does not appear in this estimation at all. In this paper, we derive an exact bound on $r_{n}$ for two-dimensional $n$ points $V$ randomly and uniformly distributed in $\mathcal{C}$ such that the graph $G\left(V, r_{n}\right)$ is $k$ connected with high probability.

We also conduct experiments to study the probability that a graph has minimum degree $k$ and has vertex connectivity $k$ simultaneously. Surprisingly, we found that, this probability is sufficiently close to 1 even $n$ is at the scale of 100 . This observation implies a simple method (by just computing the minimum vertex degree) to approximate the connectivity of a random geometry graph.

Penrose $[20,22]$ also studied the $k$-connectivity problem for $d$ dimensional points distributed in a unit-area cube using the toroidal model instead of the Euclidean model as one way to eliminate the boundary effects. He [22] showed that the hitting radius $r_{n}$ such that the graph $G\left(V, r_{n}\right)$ is $(k+1)$-connected satisfies

$$
\lim _{n \rightarrow \infty} \operatorname{Pr}\left(n \pi r_{n}^{2} \leq \ln n+k \ln \ln n-\ln k !+\alpha\right)=e^{-e^{-\alpha}} .
$$

Dette and Henze [7] studied the largest length, denoted by $r_{n, k}$ here, of the $k$ th nearest neighbor link for $n$ points drawn independently and uniformly from the $d$-dimensional unit-length cube or the $d$-dimensional unit volume sphere. They gave asymptotic result of this length according as $k<d, k=d$, or $k<d$. For unit volume cube, they use the norm $l_{\infty}$ instead of the Euclidean norm $l_{2}$. For the unit volume sphere, their result implies that, when $d=2$ and $k>2$,

$$
\begin{array}{r}
\lim _{n \rightarrow \infty} \operatorname{Pr}\left(n \pi r_{n, k}^{2} \leq\right. \\
-\ln n+(2 k-3) \ln \ln n-2 \ln (k-1) ! \\
-2(k-2) \ln 2+\ln \pi+2 \alpha)=e^{-e^{-\alpha}} .
\end{array}
$$

Notice that, Penrose [22] had showed that when the domain is a unit-area square, the probability that a random geometry graph $G\left(V, r_{n}\right)$ is $k$-connected and has minimum vertex degree $k$ goes to 1 as $n$ goes to infinity. Consequently, we can conjecture that the transmission radius $r_{n}$ such that the graph $G\left(V, r_{n}\right)$ is $k$-connected with high probability satisfies $n \pi r_{n}^{2} \simeq \ln n+(2 k-3) \ln \ln n-$ $2 \ln (k-1) !+2 \alpha$. We will prove this later.

Topology control for wireless ad hoc networks has draw considerable attentions recently $[1,13,14,15,25,26,32]$. Topology control methods try to maintain a structure that can be used for efficient routing or improving the overall networking performance. Li et al. $[14,15,32]$ had proposed to use the Yao structure [34] on the unit disk graph for topology control without sacrificing too much on the energy conservation. Yao structure does not provide fault tolerance. Recently, Bahramgiri et al. [1] proposed a fault-tolerant topology control algorithm which shows how to decide the minimum transmission range of each node such that the resulted directed communication graph is $k$-connected. However, their method does not bound the node degree. Lukovszki [16] gave a method to construct a spanner that can sustain $k$-nodes or links failures for complete graph. In this paper, we present a localized method of fault-tolerant topology control based on his method and Yao structure. We show that the constructed topology is still fault tolerant but with much fewer communication links maintained and is a length spanner. In addition, we give an enhancement of Bahramgiri's method to bound the node degree for their protocol.

\section{FAULT TOLERANT DEPLOYMENT}

In this section we concentrate on the hitting radius for the $k$ connectivity for $n$ randomly and uniformly distributed points in a unit-area square $\mathcal{C}$. We build our result based on the result by Penrose [22].

For convenience, instead of the random point process $\mathcal{X}_{n}$, we consider a homogeneous Poisson point process of rate $n$, denoted by $\mathcal{P}_{n}$, on a unit-area square $\mathcal{C}$. Same as [22], we let $\mathcal{E}(k, n, r)$ denote the expected number of points of $\mathcal{P}_{n}$ with degree $k$ in a graph of $G\left(\mathcal{P}_{n}, r\right)$. Let $D(\mathbf{x}, r)$ be the disk centered at $\mathbf{x}$ with radius $r$. Given a point $\mathbf{x}$, let $v_{r}(\mathbf{x})$ be the area of the intersection of $D(\mathbf{x}, r)$ with the unit-area square $\mathcal{C}$. Additionally, let

$$
\phi_{n, r, k}(\mathbf{x})=\left(n \cdot v_{r}(\mathbf{x})\right)^{k} \cdot \frac{e^{-n \cdot v_{r}(\mathbf{x})}}{k !} .
$$

Here $\phi_{n, r, k}(\mathbf{x})$ is the probability that point $\mathbf{x}$ has degree $k$. Then, it was known [22] that

$$
\mathcal{E}(k, n, r)=n \int_{\mathcal{C}} \phi_{n, r, k}(\mathbf{x}) d \mathbf{x} .
$$

Then Penrose [22] (Theorem 1.2) proved that:

THEOREM 1. Let $\alpha$ be any real number. Given any metric $l_{p}$ on $\mathcal{C}$ with $1<p \leq \infty$ and any integer $k \geq 0$, and $r_{n}$ satisfying the following condition

$$
\lim _{n \rightarrow \infty} \mathcal{E}\left(k, n, r_{n}\right)=e^{-\alpha},
$$

then we have

$$
\lim _{n \rightarrow \infty} \operatorname{Pr}\left(\varrho\left(\mathcal{P}_{n}, \delta \geq k+1\right) \leq r_{n}\right)=e^{-e^{-\alpha}} .
$$

Notice that, the same theorem is true when the random point process $\mathcal{P}_{n}$ is used instead of the homogeneous Poisson point process. The remainder of this section is devoted to estimate the value $r_{n}$. Penrose [22] agreed that $r_{n}$ is not so easy to find because of the dominance of complicated boundary effects. The estimated radius $r_{n}$ also makes the graph $G\left(\mathcal{P}_{n}, r_{n}\right) k$-connected with probability $e^{-e^{-\alpha}}$ when $n$ goes to infinity since Penrose [22] proved that it is almost surely that $\varrho\left(\mathcal{X}_{n}, \kappa \geq k\right)=\varrho\left(\mathcal{X}_{n}, \delta \geq k\right)$ and $\varrho\left(\mathcal{P}_{n}, \kappa \geq k\right)=\varrho\left(\mathcal{P}_{n}, \delta \geq k\right)$ as $n$ goes infinity.

\subsection{Lower Bound}

We first study the asymptotic lower bound for the hitting radius $r_{n}$ for the $(k+1)$-connectivity.

Obviously, $v_{r}(\mathbf{x}) \leq \pi r^{2}$ for any point $\mathbf{x}$ inside the unit-area square $\mathcal{C}$. Since $\phi_{n, r, k}(\mathbf{x})$ is a monotone increasing function of $v_{r}(\mathbf{x})$

$$
\phi_{n, r, k}(\mathbf{x})=\left(n \cdot v_{r}(\mathbf{x})\right)^{k} \frac{e^{-n \cdot v_{r}(\mathbf{x})}}{k !}<\left(n \cdot \pi r^{2}\right)^{k} \frac{e^{-n \cdot \pi r^{2}}}{k !}
$$

We then bound $\mathcal{E}(k, n, r)$ as follows.

$$
\mathcal{E}(k, n, r)=n \int_{\mathcal{C}} \phi_{n, r, k}(\mathbf{x}) d \mathbf{x}<n\left(n \cdot \pi r^{2}\right)^{k} \frac{e^{-n \cdot \pi r^{2}}}{k !} .
$$

Notice that if we use $\pi r^{2}$ for $v_{r}(\mathbf{x})$ instead of the actual area $v_{r}(\mathbf{x})$, the computed radius $r$ is less than the actual required radius. This is because $v_{r}(\mathbf{x})<\pi r^{2}$ for point $\mathbf{x}$ near the boundary of the square. Thus the probability that there is at least $k$ neighbors within distance $r$ of point $\mathbf{x}$ is increased when we use $\pi r^{2}$ for $v_{r}(\mathbf{x})$ for point $\mathrm{x}$ near the boundary. To remedy the approximated area $\pi r^{2}$, the actual value $r$ should be larger than the computed one. 
We estimate $r$ when $v_{r}(\mathbf{x})=\pi r^{2}$ is used as the area measurement. Let $y=\pi r^{2}$. From $\lim _{n \rightarrow \infty} \mathcal{E}\left(k, n, r_{n}\right)=e^{-\alpha}$, we have $e^{-\alpha}=\lim _{n \rightarrow \infty} n(n \cdot y)^{k} \frac{e^{-n \cdot y}}{k !}$. We will relax the condition by ignoring the condition of $n$ going infinity. In other words, we consider that

$$
e^{-\alpha}=n(n \cdot y)^{k} \frac{e^{-n \cdot y}}{k !} .
$$

It implies that, by taking $\ln$ on both sides,

$$
-\alpha=\ln n+k \ln n+k \ln y-n y-\ln (k !) .
$$

Thus, $-k \ln y+n y=(k+1) \ln n-\ln (k !)+\alpha$. Dividing both side by $k$, we have

$$
\frac{n}{k} y-\ln y=\frac{k+1}{k} \ln n-\frac{1}{k} \ln (k !)+\frac{\alpha}{k} .
$$

Let $z=\frac{n}{k} y$. Then, $\ln y=\ln z+\ln k-\ln n$. Then

$$
\begin{aligned}
z-\ln z & =\ln k-\ln n+\frac{k+1}{k} \ln n-\frac{1}{k} \ln (k !)+\frac{\alpha}{k} \\
& =\frac{1}{k} \ln n+\ln k-\frac{1}{k} \ln (k !)+\frac{\alpha}{k} .
\end{aligned}
$$

Notice that if $z=\ln z+t$, then $z>t+\ln t$, where $t>0$. Thus,

$$
\begin{aligned}
z & >\frac{1}{k} \ln n+\ln k-\frac{1}{k} \ln (k !)+\frac{\alpha}{k} \\
& +\ln \left(\frac{1}{k} \ln n+\ln k-\frac{1}{k} \ln (k !)+\frac{\alpha}{k}\right) \\
& >\frac{1}{k} \ln n+\ln k-\frac{1}{k} \ln (k !)+\frac{\alpha}{k}+\ln \left(\frac{1}{k} \ln n\right) .
\end{aligned}
$$

Consequently, by substituting back $z=\frac{n}{k} \pi r^{2}$, we have

$$
\frac{n}{k} \pi r^{2}>\frac{\ln n}{k}+\ln k-\frac{1}{k} \ln (k !)+\frac{\alpha}{k}-\ln k+\ln \ln n,
$$

which implies that

$$
n \pi r^{2}>\ln n+k \ln \ln n-\ln k !+\alpha .
$$

Notice that the function $(n \cdot y)^{k} \frac{e^{-n \cdot y}}{k !}$ achieves the maximum value when $y=\frac{k}{n}$. It is monotone decreasing for $y>\frac{k}{n}$ and monotone increasing for $y<\frac{k}{n}$. We always assume that $k$ is a fixed constant throughout this paper. Then we have the following theorem.

THEOREM 2. Given $n$ wireless nodes $V$ randomly and uniformly distributed in a unit-area square. If we want the graph $G\left(V, r_{n}\right)$ to be $(k+1)$-connected with probability at least $e^{-e^{-\alpha}}$, the transmission radius $r_{n}$ satisfies

$$
n \pi r^{2}>\ln n+k \ln \ln n-\ln k !+\alpha .
$$

Notice that, for the toroidal model, Penrose [22] gave the same exact bound for $r_{n}$ such that the graph is guaranteed to be $(k+$ 1)-connected asymptotically. Moreover, the result by Gupta and Kumar [11] and the result by Penrose [20] is just a special case when $k=0$, if this bound is tight. Notice that, in our analysis, we implicitly assume that $k>0$.

\subsection{Upper Bound}

We showed that if we want the network $G\left(V, r_{n}\right)$ to be $(k+1)$ connected with probability at least $e^{-e^{-\alpha}}$, we have to set the transmission radius $r_{n}$ satisfying inequality (1) for $n$ points randomly and uniformly distributed in a unit-area square. In this section, we continue to study the upper bound of the transmission radius to achieve the same $(k+1)$-connectivity. The estimated upper bound is different from the lower bound even asymptotically. Again, we derive the upper bound from the equation $n \int_{\mathcal{C}} \phi_{n, r, k}(\mathbf{x}) d \mathbf{x}=e^{-\alpha}$.

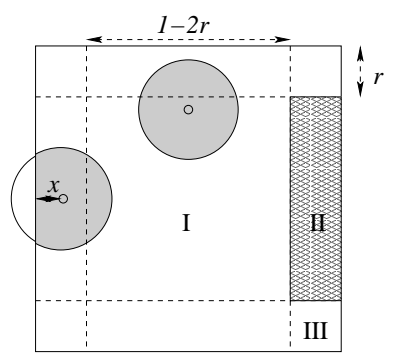

Figure 1: The area $v_{r}(\mathbf{x})$ for a point $\mathbf{x}$.

We partition the unit square to three regions: the region $\mathrm{I}$ is $[-0.5+r, 0.5-r] \times[-0.5+r, 0.5-r]$, the region III is four corners, and the remaining is the region II. See Figure 1. We compute the area $v_{r}(\mathbf{x})$ for point $\mathbf{x}$ located in these three regions separately. Obviously, for any $\mathbf{x}$ in region $\mathrm{I}, v_{r}(\mathbf{x})=\pi r^{2}$. For a point $\mathbf{x}$ in region II, assume its distance to the boundary of $\mathcal{C}$ is $x$, then the area

$$
v_{r}(\mathbf{x})=\pi r^{2}-r^{2} \cos ^{-1}\left(\frac{x}{r}\right)+x \sqrt{r^{2}-x^{2}} .
$$

Here $0 \leq x \leq r$. Assume $x=r \cos \theta$, where $0 \leq \theta \leq \pi / 2$. Then $v_{r}(\mathbf{x})=r^{2}(\bar{\pi}-\theta+\sin \theta \cos \theta)$. It is easy to show that

$$
\frac{\pi r^{2}}{2}(1+\cos \theta) \leq r^{2}(\pi-\theta+\sin \theta \cos \theta) \leq \frac{\pi r^{2}}{2}+2 r^{2} \cos \theta
$$

By substituting $x=r \cos \theta$, we bound $v_{r}(\mathbf{x})$ as follows

$$
\frac{\pi r^{2}}{2}+\frac{\pi r}{2} \cdot x \leq v_{r}(\mathbf{x}) \leq \frac{\pi r^{2}}{2}+2 r \cdot x .
$$

Let $r^{\star}$ be the solution of $n \int_{\mathcal{C}} \phi_{n, r, k}(\mathbf{x}) d \mathbf{x}=e^{-\alpha}$. Let $\Omega$ be any subregion of $\mathcal{C}$. Let $w(\mathbf{x})$ be any function such that $w(\mathbf{x}) \leq v(\mathbf{x})$ and is monotone increasing of $r$. Let $\varphi_{n, r, k}(\mathbf{x})=(n \cdot w(\mathbf{x}))^{k}$. $\frac{e^{-n \cdot w(\mathbf{x})}}{k !}$. Thus, $\varphi_{n, r, k}(\mathbf{x}) \leq \phi_{n, r, k}(\mathbf{x})$. Let $r^{\prime}$ be the solution of $n \int_{\Omega} \varphi_{n, r, k}(\mathbf{x}) d \mathbf{x}=e^{-\alpha}$. Then $r^{\star} \leq r^{\prime}$. This is because $w(x)$, $v_{r}(x)$ are monotone increasing functions of $r$, and $(n y)^{k} \frac{e^{-n y}}{k !}$ is monotone increasing function when $y \leq k / n$. Thus, to bound the transmission radius $r$ from above so that the graph $G(V, r)$ is $(k+1)$-connected, we use the lower bound of $v_{r}(\mathbf{x})$ and we also only compute the integral for region I and region II. Notice,

$$
\begin{aligned}
& \int_{\mathcal{C}}\left(n v_{r}(\mathbf{x})\right)^{k} \frac{e^{-n v_{r}(\mathbf{x})}}{k !} d \mathbf{x} \\
> & \int_{I}\left(n v_{r}(\mathbf{x})\right)^{k} \frac{e^{-n v_{r}(\mathbf{x})}}{k !} d \mathbf{x}+\int_{I I}\left(n v_{r}(\mathbf{x})\right)^{k} \frac{e^{-n v_{r}(\mathbf{x})}}{k !} d \mathbf{x}
\end{aligned}
$$

Obviously, for region I, we have

$$
\int_{I}\left(n v_{r}(\mathbf{x})\right)^{k} \frac{e^{-n v_{r}(\mathbf{x})}}{k !} d \mathbf{x}=\left(n \cdot \pi r^{2}\right)^{k} \cdot \frac{e^{-n \cdot \pi r^{2}}}{k !} \cdot(1-2 r)^{2} .
$$

The integral over region II is 4 times of the integral over the rectangle region near the boundary, where the length of the rectangle is $1-2 r$ and the width is $r$. Let the distance of a point $\mathbf{x}$ to the 
boundary be $x$. Let $y=\frac{\pi r^{2}}{2}+\frac{\pi r}{2} x$. Notice $v_{r}(\mathbf{x})>y$. We have

$$
\begin{aligned}
& \int_{I I}\left(n \cdot v_{r}(\mathbf{x})\right)^{k} \frac{e^{-n \cdot v_{r}(\mathbf{x})}}{k !} d \mathbf{x} \\
& =4(1-2 r) \int_{x=0}^{r}\left(n v_{r}(x)\right)^{k} \frac{e^{-n v_{r}(x)}}{k !} d x \\
& >\frac{8(1-2 r)}{\pi \cdot k ! \cdot r} \int_{y=\frac{\pi r^{2}}{2}}^{\pi r^{2}}(n y)^{k} e^{-n y} d y \\
& =\frac{8(1-2 r)}{n \pi r}\left(e^{-t / 2} \sum_{j=0}^{k} \frac{t^{j}}{j ! 2^{j}}-e^{-t} \sum_{j=0}^{k} \frac{t^{j}}{j !}\right) .
\end{aligned}
$$

Here $t=n \pi r^{2}$. The last equation comes from $\int z^{k} e^{-z} d z=$ $-e^{-z} k ! \sum_{j=0}^{k} \frac{z^{j}}{j !}$. Then the transmission radius $\left.\varrho\left(\mathcal{P}_{n}, \kappa \geq k\right)\right)$ is bounded from above by the solution of the following equation.

$$
\begin{aligned}
e^{-\alpha} & =n \cdot t^{k} \frac{e^{-t}}{k !} \cdot(1-2 r)^{2}+\frac{8(1-2 r)}{\pi r}\left(e^{-t / 2} \sum_{j=0}^{k} \frac{(t / 2)^{j}}{j !}-e^{-t} \sum_{j=0}^{k} \frac{t^{j}}{j !}\right) \\
& <n \cdot t^{k} \frac{e^{-t}}{k !}+\frac{8}{\pi r} k \cdot e^{-t / 2} \frac{(t / 2)^{k}}{k !} .
\end{aligned}
$$

The inequality comes from $e^{-t / 2} \frac{(t / 2)^{j}}{j !}<e^{-t / 2} \frac{(t / 2)^{j+1}}{(j+1) !}$ for $j<$ $t / 2$ assuming that $k<t / 2$. From our lower bound analysis, $t=$ $n \pi r^{2} \geq \ln n$ asymptotically. The rest of the section is then devoted to approximate $r$.

Let $A=n \cdot t^{k} \frac{e^{-t}}{k !}$ and $B=\frac{8}{\pi r} k \cdot e^{-t / 2} \frac{(t / 2)^{k}}{k !}$. Thus, $\frac{B}{A}=$ $\frac{8 k e^{t / 2}}{2^{k} n \pi r}=\frac{8 k}{\sqrt{\pi}} \frac{e^{t / 2}}{\sqrt{n t}}$. Then, by taking ln on both sides of the inequality, we have

$$
\begin{aligned}
-\alpha & <\ln A+\ln \left(1+\frac{B}{A}\right) \\
& =\ln n+k \ln t-t-\ln k !+\ln \left(1+\frac{8 k}{2^{k} \sqrt{\pi}} \frac{e^{t / 2}}{\sqrt{n t}}\right) .
\end{aligned}
$$

Thus, we have

$$
t<\ln n+k \ln t-\ln k !+\alpha+\ln \left(1+\frac{8 k e^{t / 2}}{2^{k} \sqrt{\pi n t}}\right) .
$$

Notice that $\ln (1+x)<x$ for any $1>x>0$ and $\ln (1+x) \simeq$ $\ln x$ for $x$ sufficiently larger than one. We solve inequality (2) by recursion as follows. First let $t_{1}=\ln n-\ln k !+\alpha$ as the initial solution. It is easy to show that $\frac{B}{A}=\frac{8 k}{\sqrt{\pi}} \frac{e^{t_{1} / 2}}{\sqrt{n t_{1}}} \ll 1$. Thus, we can estimate the solution by substituting $t_{1}$ to inequality (2)

$$
t_{2}<\ln n+k \ln t_{1}-\ln k !+\alpha+\ln \left(1+\frac{8 k}{2^{k} \sqrt{\pi}} \frac{e^{t_{1} / 2}}{\sqrt{n t_{1}}}\right) .
$$

When $n$ is large enough, we have $t_{2} \simeq \ln n+k \ln \ln n-\ln k !+\alpha$. In this situation, however, we have $\frac{B}{A}=\frac{8 k}{2^{k} \sqrt{\pi}} \frac{e^{t_{2} / 2}}{\sqrt{n t_{2}}}=\frac{8 k}{2^{k} \sqrt{\pi}} \sqrt{\frac{(\ln n)^{k} e^{\alpha}}{k ! \cdot t_{2}}}$ goes to infinity when $n$ goes to infinity. Thus, by substituting $t_{2}=$ $\ln n+k \ln \ln n-\ln k !+\alpha$ to inequality (2), we have the third estimation of the solution as follows

$$
\begin{aligned}
t_{3} & <\ln n+k \ln t_{2}-\ln k !+\alpha+\ln \left(1+\frac{8 k e^{t_{2} / 2}}{2^{k} \sqrt{\pi n t_{2}}}\right) \\
& \simeq \ln n+k \ln t_{2}-\ln k !+\alpha+\ln \frac{8 k e^{t_{2} / 2}}{2^{k} \sqrt{\pi n t_{2}}} \\
& =\ln n-\frac{3}{2} \ln k !+\frac{3}{2} \alpha+\frac{1}{2} k \ln \ln n+\left(k-\frac{1}{2}\right) \ln t_{2}+\ln \frac{8 k}{2^{k} \sqrt{\pi}} .
\end{aligned}
$$

Notice that

$$
\begin{aligned}
\ln t_{2} & =\ln (\ln n+k \ln \ln n-\ln k !+\alpha) \\
& =\ln \ln n+\ln \left(1+\frac{k \ln \ln n-\ln k !+\alpha}{\ln n}\right) \\
& <\ln \ln n+\frac{k \ln \ln n-\ln k !+\alpha}{\ln n} .
\end{aligned}
$$

Thus, we have the third estimation $t_{3}$ as $\ln n+\frac{1}{2}(3 k-1) \ln \ln n-$ $\frac{3}{2} \ln k !+\frac{3}{2} \alpha+\ln \frac{8 k}{2^{k} \sqrt{\pi}}$. We can continue to substitute $t_{3}$ to get a more accurate solution $t_{4}$ and so on. It is easy to show that the final solution of $t$ is bounded by solution of the following equality when $n$ goes to infinity

$$
t=\ln n+k \ln t-\ln k !+\alpha+\ln \left(\frac{8 k}{2^{k} \sqrt{\pi}} \frac{e^{t / 2}}{\sqrt{n t}}\right)
$$

This implies that

$$
t=\ln n-2 \ln k !+2 \alpha+2 \ln \frac{8 k}{\sqrt{\pi}}+(2 k-1) \ln t .
$$

Thus we can bound $t$ by the following approximation, when $n$ goes to infinity

$$
t=\ln n+(2 k-1) \ln \ln n-2 \ln k !+2 \alpha+2 \ln \frac{8 k}{2^{k} \sqrt{\pi}} .
$$

Consequently, we have

THEOREM 3. Given $n$ wireless nodes $V$ randomly and uniformly distributed in a unit-area square. If we set the transmission radius $r_{n}$ to satisfy that

$$
n \pi r^{2}>\ln n+(2 k-1) \ln \ln n-2 \ln k !+2 \alpha+2 \ln \frac{8 k}{2^{k} \sqrt{\pi}},
$$

then the graph $G\left(V, r_{n}\right)$ is $(k+1)$-connected with probability at least $e^{-e^{-\alpha}}$ when $n$ goes to infinity.

Obviously, if $\alpha \rightarrow \infty$, then $e^{-e^{-\alpha}} \rightarrow 1$. For example, if we set $\alpha=\ln \ln n$, i.e., want the graph $G\left(V, r_{n}\right)$ to be $(k+1)$-connected with probability at least $e^{-1 / \ln n}>1-\frac{1}{\ln n}$, we have to set the transmission radius $r_{n}$ that satisfies

$$
n \pi r^{2}>\ln n+(2 k+1) \ln \ln n-2 \ln k !+2 \ln \frac{8 k}{2^{k} \sqrt{\pi}} .
$$

If we want to the graph $G\left(V, r_{n}\right)$ to be $(k+1)$-connected with probability at least $e^{-1 / n}>1-\frac{1}{n}$, we have to set the transmission radius $r_{n}$ satisfying

$$
n \pi r^{2}>3 \ln n+(2 k-1) \ln \ln n-2 \ln k !+2 \ln \frac{8 k}{2^{k} \sqrt{\pi}} .
$$

Additionally, if $\alpha \rightarrow-\infty$, then $e^{-e^{-\alpha}} \rightarrow 0$. Then it implies that the graph $G\left(V, r_{n}\right)$ will be $(k+1)$-connected with very low probability if this bound of the hitting radius is tight.

Notice that the above analysis of the asymptotic upper bound of the transmission radius can also be used to derive a tighter lower bound on the transmission radius. We use the fact that $\frac{\pi r^{2}}{2}+\frac{\pi r}{2}$. $x \leq v_{r}(\mathbf{x})$ to derive the upper bound of the transmission radius. To analyze the lower bound, we have to use the fact that $v_{r}(\mathbf{x}) \leq$ $\frac{\pi r^{2}}{2}+2 r \cdot x$ to estimate the area $v_{r}(\mathbf{x})$ for point $\mathbf{x}$ near the boundary. In addition, we have to compute the integral in all three regions. To simplify the analysis, for point $\mathbf{x}$ in region III, we also use $v_{r}(\mathbf{x}) \leq$ $\frac{\pi r^{2}}{2}+2 r \cdot x$ to estimate the area $v_{r}(\mathbf{x})$. Then similar to the above 
analysis of upper bound, the lower bound on $t$ is at least the solution of the following equation

$$
e^{-\alpha}=n \cdot t^{k} \frac{e^{-t}}{k !} \cdot(1-2 r)^{2}+\frac{2}{r}\left(e^{-\frac{t}{2}} \sum_{j=0}^{k} \frac{t^{j}}{2^{j} j !}-e^{-t} \sum_{j=0}^{k} \frac{t^{j}}{j !}\right) .
$$

By tedious computing, we get the asymptotic lower bound as

$$
t>\ln n+(2 k-1) \ln \ln n-2 \ln k !+2 \alpha .
$$

REMARK: Although we have computed the lower and upper bounds for the transmission range $r_{n}$ such that the graph $G\left(V, r_{n}\right)$ is $(k+1)$-connected with probability at least $e^{-e^{-\alpha}}$, these bounds hold only when $n$ goes to infinity and $k$ is assumed to be a constant. Our simulations show that $n$ should be larger than 500 if $k \leq 2$ and $\alpha=\log n$; and $n$ should be larger than 2500 if $k \leq 2$ and $\alpha=\log \log n$. When $n$ is a small number (especially when $n$ is comparable with $k$ !), our bounds do not hold anymore. This observation is witnessed by our experimental results (there is a considerably large difference between theoretical bound and the actual transmission range).

\section{TOPOLOGY CONTROL FOR FAULT TOLERANCE}

In this section, we study how to control the network topology given a $n$ nodes network that is already $k$ fault tolerant. After selecting the hitting radius for the $k$-connectivity, we can model the network topology as a unit disk graph (UDG) by scaling the radius to one unit. A unit disk graph is the graph in which two nodes are connected if their distance is not more than one unit.

Due to the nodes' limited resource in wireless ad hoc networks, the scalability is crucial for network operations. One effective approach is to maintain only a linear number of links using a localized construction method. However, this sparseness of the constructed network topology should not compromise on the fault tolerance and compromise too much on the power consumptions for both unicast and broadcast/multicast communications. We are interested in constructing a sparse network topology efficiently for a set of static wireless nodes such that every unicast route in the constructed network topology is power efficient, in addition to be $k$ fault tolerant. Here a route is power efficient for unicasting if its power consumption is no more than a constant factor of the minimum power needed to connect the source and the destination. A network topology is said to be power efficient if there is a power efficient route to connect any two nodes in this topology.

In the most common power-attenuation model, the signal power falls as $\frac{1}{r^{\beta}}$, where $r$ is the distance from the transmitter antenna and $\beta$ is a constant between 2 and 5 dependent on the wireless transmission environment. This is called path loss. For simplicity, we only consider the path loss of the signal. Thus, the power needed to support a link $u v$ is $\|u v\|^{\beta}$, where $\|u v\|$ is the Euclidean distance between $u$ and $v$.

Lukovszki [16] gave a method to construct a spanner that can sustain $k$-nodes or links failures for complete graph. Our topology control method is based on this method and the following Yao structure [34]. The Yao graph over a (directed) graph $G$ with an integer parameter $p \geq 6$, denoted by $\overrightarrow{Y G}_{p}(G)$, is defined as follows. At each node $u$, any $p$ equal-separated rays originated at $u$ define $p$ equal cones. In each cone, choose the shortest (directed) edge $u v \in G$, if there is any, and add a directed link $\overrightarrow{u v}$. Ties are broken arbitrarily. Let $Y G_{p}(G)$ be the undirected graph by ignoring the direction of each link in $\overrightarrow{Y G}_{p}(G)$. See Figure 2 for an illustration of selecting edges incident on $u$ in the Yao graph.

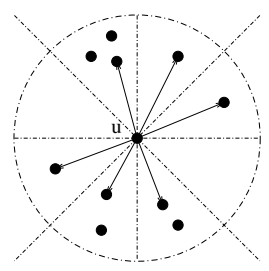

Figure 2: The narrow regions are defined by 8 equal cones. The closest node in each cone is a neighbor of $u$.

Li et al. $[14,15,32]$ had proposed to use the Yao structure on the unit disk graph for topology control without sacrificing too much on the energy conservation. Some researchers used a similar construction named $\theta$-graph [16]. The difference is that, in each cone, it chooses the edge which has the shortest projection on the axis of the cone instead of the shortest edge. Here the axis of a cone is the angular bisector of the cone. For more detail, please refer to [16]. It is obvious that the Yao structure does not sustain $k$ faults in a neighborhood of any node since each node only has at most $p$ neighbors and one neighbor selected in each cone at most. However, we can modify the Yao structure as follows such that the structure is $k$-fault tolerant.

Each node $u$ defines any $p$ equal-separated rays originated at $u$, thus defines $p$ equal cones, where $p>6$. In each cone, node $u$ chooses the $k+1$ closest nodes in that cone, if there is any, and add directed links from $u$ to these nodes. Ties are broken arbitrarily. Let $Y_{p, k+1}$ be the final topology formed by all nodes.

THEOREM 4. The structure $Y_{p, k+1}$ can sustain $k$ nodes faults if original unit disk graph is $k$ node faults tolerant.

PROOF. For simplicity, assume that all $k$ fault nodes $v_{1}, v_{2}, \cdots$, $v_{k}$ are neighbors of a node $u$. We show that the remaining graph of $Y_{p, k+1}$ (removed of nodes $v_{1}, v_{2}, \cdots, v_{k}$ and all links incident on them) is still connected.

Notice that the original unit disk graph is $k$ node faults tolerant. Thus, the degree of each node is at least $k+1$. Additionally, with the $k$ fault nodes $v_{1}, v_{2}, \cdots, v_{k}$ removed, there is still a path in UDG to connect any pair of remaining nodes. Assume that the path uses node $u$ and have a link $u w$. We will prove by induction that there is a path in the remaining graph to connect $u$ and $w$.

If $u w$ has the smallest distance among all pair of nodes, then $u w$ must be in $Y_{p, k+1}$, thus in the remaining graph.

Assume the statement is true for node pair whose distance is the $r$ th shortest. Consider $u w$ with the $(r+1)$ th shortest length.

If $w$ is one of the $k+1$ closest nodes to $u$ in some cone, then link $u w$ remains in the remaining graph. Otherwise, for the cone in which node $w$ resides, there must have other $k+1$ nodes which are closer to $u$ than $w$ and they are connected by $u$ in $Y_{p, k+1}$. Since we only have $k$ failure nodes, at least one of the links of $Y_{p, k+1}$ in that cone will survive, say link $u x$. It is easy to show that $\|x w\|<$ $\|u w\|<1$. Then link $u w$ can be replaced by link $u x$ and a path from $x$ to $w$ by induction. This finishes the proof.

Notice that for the case where the nodes removed are not all neighbors of the same node, the induction proof also holds. Induction is based on all pair of nodes.

Our techniques of constructing $k$-connected subgraph of UDG (assuming UDG is already $k$-connected here) can be applied to a more general graph $G$ if there is an embedding, denoted by $E(G)$, 
of $G$ in the plane such that there is an edge in $E(G)$ iff their distance is not more than one unit. Notice that here an embedding of $G$ in the plane is to assign each vertex a two-dimensional position.

We then show that the above structure approximates the original unit disk graph well. More specifically, we will show that it is a spanner even with $k$ fault nodes. Let $\Pi_{G}(u, v)$ be the shortest path connecting $u$ and $v$ in a weighted graph $G$, and $\left\|\Pi_{G}(u, v)\right\|$ be the length of $\Pi_{G}(u, v)$. Then a graph $G$ is a $t$-spanner of a graph $H$ if $V(G)=V(H)$ and, for any two nodes $u$ and $v$ of $V(H)$, $\left\|\Pi_{G}(u, v)\right\| \leq t\left\|\Pi_{H}(u, v)\right\|$. With $H$ understood, we also call $t$ the length stretch factor of the spanner $G$.

Let $\varrho_{G}(u, v)$ be the path found by a unicasting routing method $\varrho$ from node $u$ to $v$ in a weighted graph $G$, and $\left\|\varrho_{G}(u, v)\right\|$ be the length of the path. The spanning ratio achieved by a routing method $\varrho$ is defined as $\max _{u, v}\left\|\varrho_{G}(u, v)\right\| /\|u v\|$. Notice that the spanning ratio achieved by a specific routing method could be much larger than the spanning ratio of the underlying structure. Nonetheless, a structure with a small spanning ratio is necessary for some routing method to possibly perform well.

THEOREM 5. The structure $Y_{p, k+1}$ is a length spanner even with $k$ nodes faults.

PROOF. To prove the length spanner property, it is easy to show that we only have to prove each pair of nodes $u$ and $w$ with $\|u w\| \leq$ 1 is approximated by a path with length no more than a constant factor, say $\beta$, of $\|u w\|$. The proof is similar to Theorem 4: we prove it by induction on the length of $\|u w\|$. Follow the proof of Theorem 4, we only have to show that

$$
\|u x\|+\beta\|x w\| \leq \beta\|u w\|
$$

for any node $x$ with $\|u x\|<\|u w\|$ and $x$ lies in the same cone as $w$ does. Obviously, we need to set

$$
\beta=\max _{\forall x,\|u x\|<\|u w\|} \frac{\|u x\|}{\|u w\|-\|x w\|} .
$$

Notice that $\alpha=\angle w u x<\frac{2 \pi}{p}$. Then a simple geometry reveals that $\beta=\max \frac{\cos \theta}{\cos (\theta+\alpha)}$, where $\theta=\frac{1}{2} \angle u w x \leq \frac{\pi-\alpha}{2}$. The minimum value for $\beta$ is $\frac{1}{1-2 \sin (\pi / p)}$. In other words, the spanning ratio of the remaining structure is at most $\beta$.

Due to limited power and resource of wireless nodes, wireless topologies always prefer to have bounded node degree, such that every wireless nodes only keep constant neighbors. The node degree of the structure $Y_{p, k+1}$ is at most $p(k+1)$, where $p \geq 6$. Recently, Bahramgiri et al. [1] showed how to decide the minimum transmission range of each node such that the resulted directed communication graph is $k$-connected. We can prove that their resulted graph is also a length spanner even with $k$ nodes faults (the proof is omitted here since it is similar to ours). However, their method does not bound the node degree. Figure 3(a) shows an example in which node $u$ can have as many as neighbors even after applying their method. Then we give a careful enhancement of their protocol to bound the node degree. In Bahramgiri's method, they increase the power step by step until there is no gap greater than $\alpha$ between the successive neighbors or the power reaches the maximum power. They proved that if $\alpha \leq \frac{2 \pi}{3 k}$ then the resulted graph is $k$-connected. After applying their method, we can remove some links by the following method. For a node $u$, we divide its transmission range into $\frac{4 \pi}{\alpha}$ equal cones (each cone have an angle $\alpha / 2$ ). We select only one neighbor in each cone $c$ if there is any, delete all other links. However, if for a cone $c$, one of its adjacent cones, say $b$, does not have any neighbors of $u$, we select the boundary neighbor $v$ such that $v u$ forms the smallest angle with cone $b$;

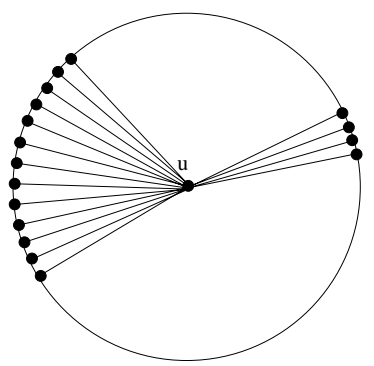

(a)

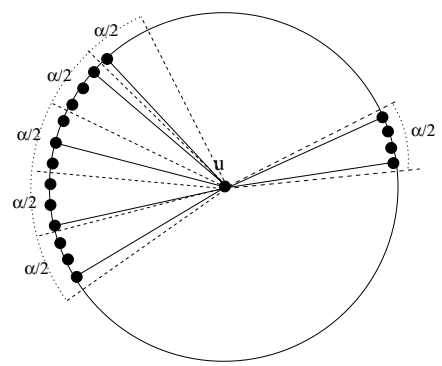

(b)
Figure 3: (a) node $u$ does not have bounded degree in graph generated by Bahramgiri's protocol; (b) new method to bound node degree for Bahramgiri's protocol.

if both adjacent cones of $c$ are empty, we select two neighbors in $c$ (close to the two boundary of cone $c$ respectively); if $c$ does not have empty adjacent cones, we can select any one of the neighbors. See Figure 3(b) for illustration. Since the gap between any two successive remaining neighbors is still not greater than $\alpha$ (except the empty cones), it is easy to show that the constructed graph is still $k$-connected if $\alpha \leq \frac{2 \pi}{3 k}$. The node degree is bounded by $\frac{2 \pi}{\frac{\alpha}{2}}=\frac{4 \pi}{\alpha}$. When $\alpha=\frac{2 \pi}{3 k}$, the node degree is bounded by $6 k$, which is almost the same as ours.

\section{EXPERIMENTS}

We had analyzed the theoretical condition for the transmission radius $r_{n}$ such that the graph $G\left(V, r_{n}\right)$ is $(k+1)$-connected with high probability. To confirm our theoretical analysis, we conduct simulations to see what is the practical value of $r_{n}$ such that the wireless network $G\left(V, r_{n}\right)$ is $(k+1)$-connected with high probability. Notice that Bettstetter [2] also conducted simulations recently to study the $k$-connectivity, minimum degree being $k$, and their relations. No explicit expression of $r$ is given in [2].

\subsection{System Model}

The geometry domain, in which the wireless nodes are distributed, is a unit square $\mathcal{C}=[-0.5,0.5] \times[-0.5,0.5]$. As shown by previous results, we know that the random point process $\mathcal{X}_{n}$ and the homogeneous Poisson point process $\mathcal{P}_{n}$ will have the same connectivity behavior asymptotically. For the simplicity of conducting simulations, we choose $n$ points that are randomly and uniformly distributed in $\mathcal{C}$. For each randomly generated point set $V$ and a transmission radius $r$, we construct the graph $G(V, r)$ in a centralized manner. To speed up the construction of $G(V, r)$, we partition the points into grids of size $r$. Thus, a point $p$ can only connect with points from at most 9 grids: one grid containing $p$ and 8 adjacent grids.

\subsection{Computing the Connectivity}

One of the major steps in conducting the simulations is to compute the connectivity of an induced unit disk graph $G\left(V, r_{n}\right)$. It is easy to test whether a graph is connected by simply checking if a spanning tree contains all $n$ nodes. To test whether the graph $G\left(V, r_{n}\right)$ is $k$-connected, we use the following observation: it is $k$-connected if and only if the minimum cut is at least $k$, which is equivalent to that the flow between any pair of nodes is at least $k$. So, given the graph $G\left(V, r_{n}\right)$, we compute the maximum flow between any pair of nodes by assigning each edge a weight one. A simpler method by using BFS to compute how many disjoint paths connecting a node $v$ to a node $u$. The time complexity of this ap- 
proach is $O\left(n^{2} m\right)$, where $m$ is the number of edges in $G(V, r)$ which could be as large as $n^{2}$. For unit-capacity flow, there is an $O\left(\min \left(m, n^{3 / 2}\right) m^{1 / 2}\right)$ time complexity algorithm [8].

\subsection{Experimental Results}

TRANSITION PHENOMENA: A graph property of $G(V, r)$ is said to satisfy a transition phenomena if there is a radius $r_{0}$ such that the graph $G(V, r)$ almost surely does not have this property when $r<r_{0}$ and the graph $G(V, r)$ almost surely has this property when $r>r_{0}$. It was already shown that the property that $G(V, r)$ has the minimum node degree $k$ satisfies a transition phenomena; additionally, the graph $G(V, r)$ is $k$-connected satisfies a transition phenomena. Our simulations shown in Figures 4 and Figures 5 confirm the theoretical results. We found that the transition becomes faster when the number of nodes increases. For testing the transition phenomena of the connectivity, we test $n=50$ and $n=100$ two cases. We test $0.1 \leq r \leq 0.9$ using interval 0.02 , i.e., we test total 40 different transmission radii. Given a transmission radius $r$ and number of nodes $n$, we generate 500 sets of random $n$ points in $\mathcal{C}$. We compute the connectivity of each graph $G(V, r)$ and summarize how many is $k$-connected for $k=1,2,3$ and 4 . For testing the transition phenomena of the min-degree, we test $n=100,200$, 300 , and 400 . Other settings are same as the test for connectivity transition.
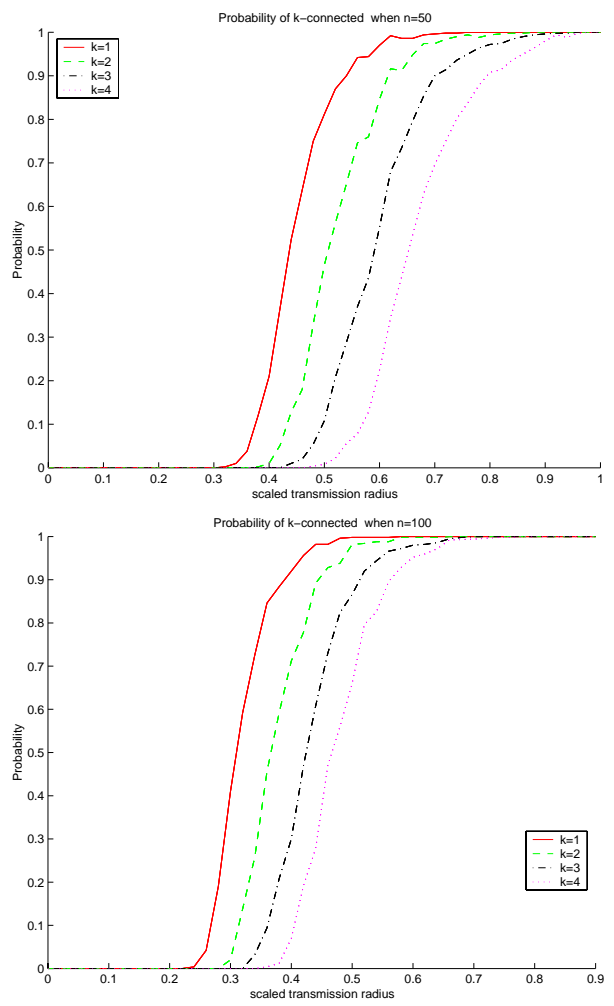

Figure 4: Transition phenomena of a graph being $k$-connected. Up figure for $n=50$ and lower figure for $n=100$.

Connectivity And Minimum DegReE: Penrose [22] showed that the hitting radius for $k$-connectivity and the hitting radius for achieving minimum degree $k$ are asymptotically same for points randomly and uniformly distributed in a unit-area square as $n$ goes infinity. We conduct extensive simulations on various number of points $n=50,100,200,300,400$ and 500. Given $n, k$, and $\alpha$, we select $r$ according to the bound given in Theorem 3 . Here the con-
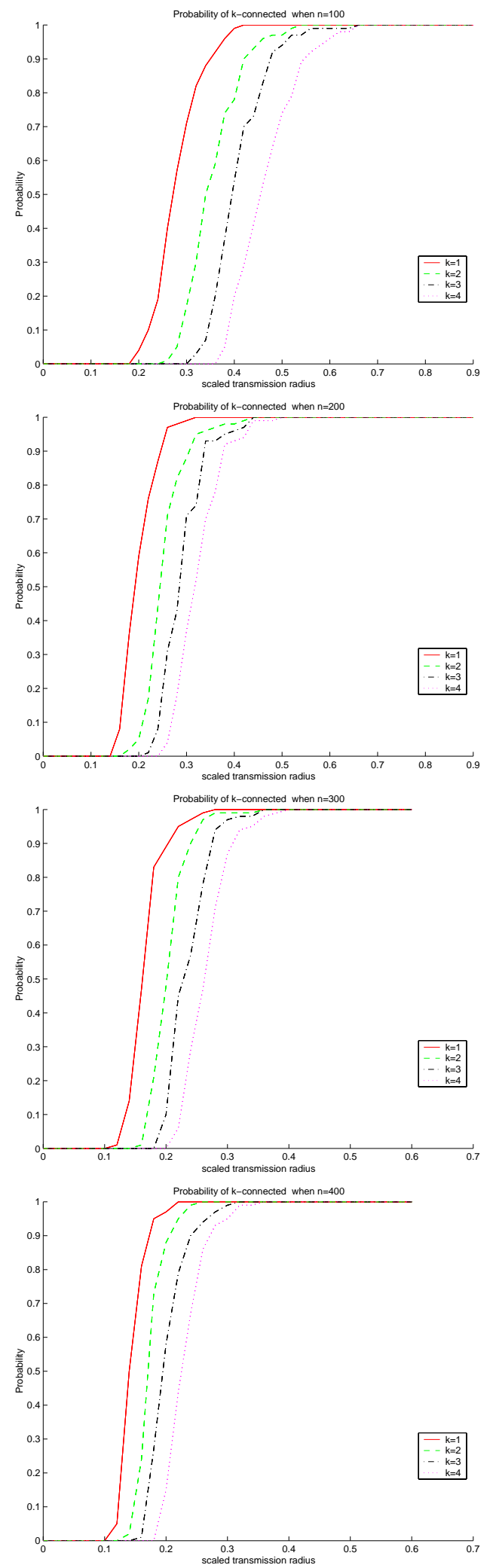

Figure 5: Transition phenomena of a graph with min-degree $k$. 
nectivity $k=1,2$ and $\alpha \in\{0, \ln \ln n, \ln n\}$. Thus, there are total 36 cases. For each case, we generate 500 random point sets. Our simulations illustrated by Figure 6 show that the probability that $G(V, r)$ is $k$-connected when its minimum degree is $k$ is already sufficiently close to one when $n$ is at the order of 50, especially when $\alpha$ is set as $\ln n$. This surprising result implies a fast method to approximate the connectivity of a graph by simply counting the minimum node degree.

Connectivity for SMAll Point Set: Theoretically, we derived an asymptotic bound of the transmission range $r_{n}$ for $n$ points randomly and uniformly distributed in a unit-area square such that the graph $G\left(V, r_{n}\right)$ is $k$-connected with certain probability. We have to admit that the result holds only when $n$ is large enough compared with $k$ !. We first conduct simulations to measure the gap between the theoretical probability of graph $G(V, r)$ being $k$-connected and the actual statistical probability of it being $k$-connected for various radius $r$. Typically, we set $n \pi r^{2}=$ $\ln n+(2 k-1) \ln \ln n-2 \ln k !+2 \alpha+2 \ln \frac{8 k}{2^{k} \sqrt{\pi}}$. Then test all 54 cases of $n=50,100,200,300,400$, and $500, k=1,2,3$, and $4, \alpha=0, \ln \ln n$, and $\ln$. The corresponding theoretical $k$ connectivity probabilities for them are $\frac{1}{e}, 1-\frac{1}{\ln n}$, and $1-\frac{1}{n}$ when $\alpha=0, \ln \ln n$, and $\ln n$ respectively. The probability is computed over 500 different random point sets. Figure 7 illustrates our simulation results.
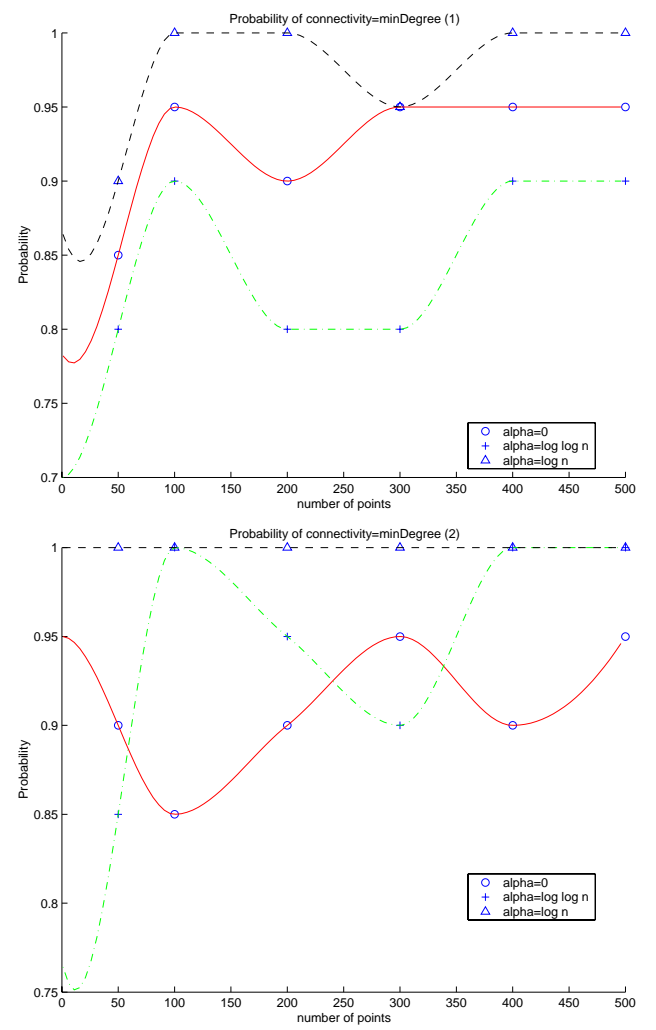

Figure 6: The probability of a graph with minimal degree $k$ is $k$-connected. Upper figure is for $k=1$ and lower figure is for $k=2$.

It is not surprised that the probability found by simulations is much lower than the theoretical analysis (denoted by the most upper curves). Notice that the theoretical range $r$ is not always monotone increasing of $k$ when $n$ is a small value. Figure 9 illustrates our simulation results for the probability that $G(V, r)$ has mini- mum degree $k$ compared with the theoretical analysis. Notice, as expected, the probability gap for min-degree is smaller than that for the $k$-connectivity.

PRACTICAL TRANSMISSION RANGES FOR $k$-CONNECTIVITY: Since the asymptotic bound of the transmission range $r_{n}$ for $n$ points randomly and uniformly distributed in a unit-area square such that the graph $G\left(V, r_{n}\right)$ is $k$-connected with certain probability holds only when $n$ is large enough compared with $k$ !, we need study what is the actual transmission range required to achieve the $k$-connectivity with certain probability. It is possible to analyze more accurately what is the theoretical requirement for $r_{n}$ when $n$ is not large enough. However, the analysis is much more complicated as we cannot omit some "constant" terms in any formula anymore. We leave this tight analysis as possible future work. Alternatively, we conduct simulations to find that practical transmission ranges when $n$ is not large enough. See Figure 8 and Figure 10. It is not surprise that the actual required range is larger than the theoretical bound. However, we found that the actual transmission range takes a similar decreasing pattern as the theoretical result when $n$ goes infinity.

\section{CONCLUSION}

We consider a large-scale of wireless ad hoc networks whose nodes are distributed in a two-dimensional unit square region. As fault-tolerance is imperative for wireless networks, we showed that, if the transmission range $r_{n}$ satisfies $n \pi \cdot r_{n}^{2} \geq \ln n+(2 k-$ 1) $\ln \ln n-2 \ln k !+2 \alpha$, the graph $G\left(V, r_{n}\right)$ is $(k+1)$-connected with probability at least $e^{-e^{-\alpha}}$. When $n$ is small (especially when $n$ is comparable with $k$ !), our bounds do not hold anymore. Our simulations showed that $n$ should be larger than 500 if $k \leq 2$ and $\alpha=\log n$; and $n$ should be larger than 2500 if $k \leq 2$ and $\alpha=\log \log n$. Our result holds also in mobile networks when the movement of nodes are also random. We leave an accurate theoretical analysis of the transmission range to achieve $k$-connectivity, minimum degree $k$ when number of nodes $n$ is small as open questions.

We then presented a localized method to control the network topology given a $(k+1)$-faults tolerant deployment $G\left(V, r_{n}\right)$ of wireless nodes such that the resulting topology $Y_{p, k+1}$ is still fault tolerant but with much fewer communication links maintained. We show that $Y_{p, k+1}$ has only linear number of links and is a length spanner. Finally, we conducted extensive simulations to study the relations between the minimum node degree and the connectivity of the induced unit disk graphs. Practical transmission ranges were also studied by simulations when $n$ is not a large integer. We found that, although it is different from the theoretical analysis when $n$ is small, it has the same decreasing pattern as our theoretical analysis.

Notice that, we assumed that the wireless nodes are generated by random point process, or Poisson point process. In practical applications, the wireless nodes could have some other estimated distributions such as the inhomogeneous Poisson point process. This is much more complicated than the cases studied by known previous results. We leave this as possible future work.

Nodes in wireless ad hoc networks may become inactive or unavailable due to either internal breakdown or being in the sleeping state. The inactive nodes cannot take part in routing/relaying and thus may affect the connectivity. Recently, Yi et al. [35] modeled the availability of the nodes by a Bernoulli model, in which nodes are active (or available) independently with probability $p$ $(0<p \leq 1)$. They showed that if all nodes have a maximum transmission radius $r_{n}=\sqrt{\frac{\operatorname{lnn}+c}{\pi p n}}$ for some constant $c$, then the total number of isolated nodes is asymptotically Poisson with mean 

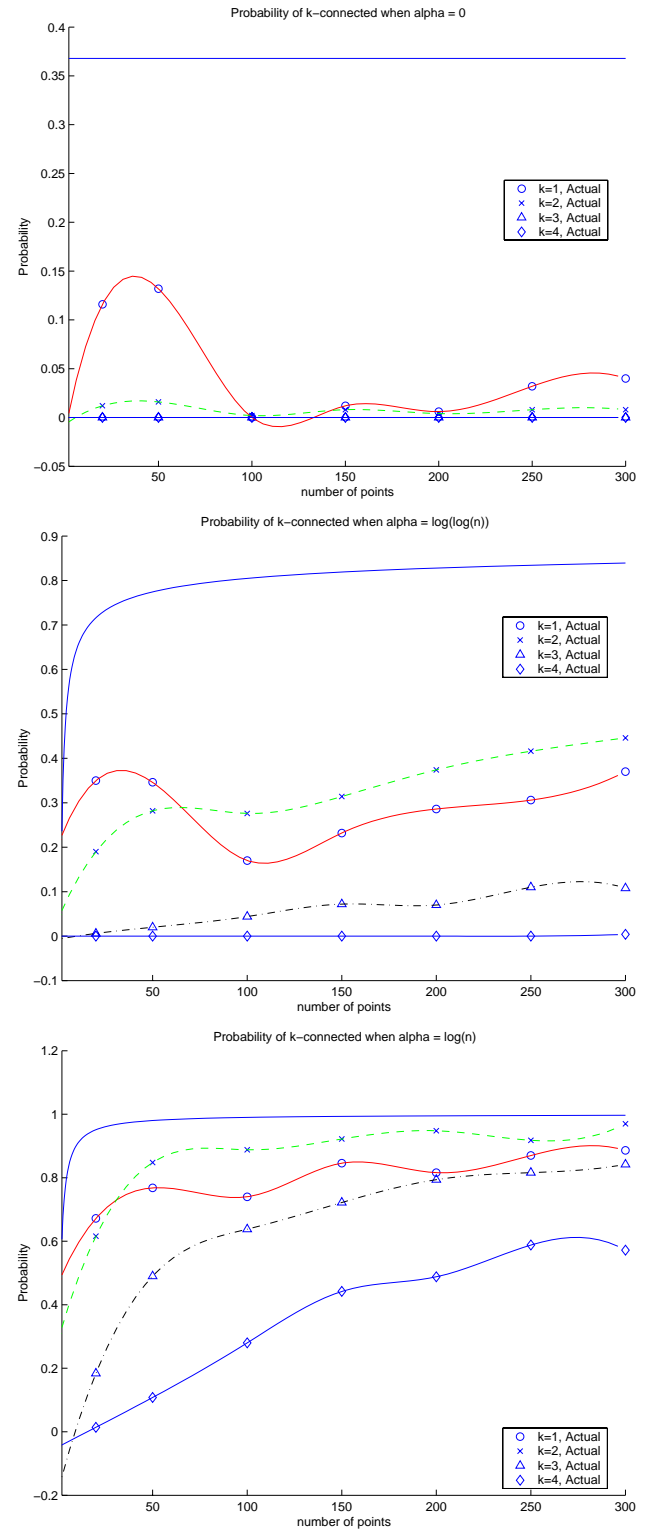

Figure 7: Probability that $G(V, r)$ is $k$-connected for theoretical $r$.

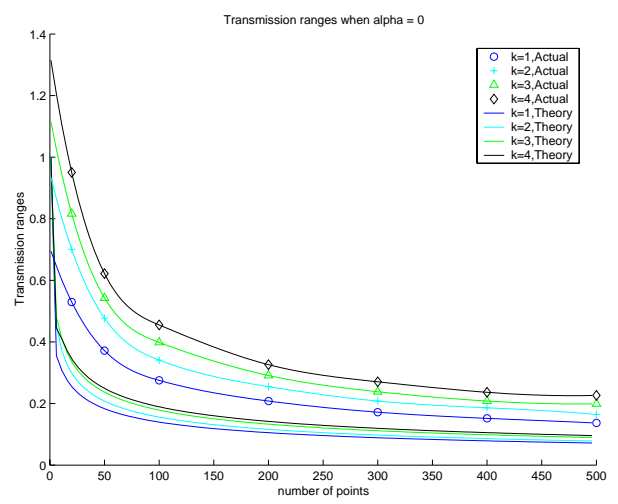

Figure 8: Practical range that $G(V, r)$ is $k$-connected with probability $1 / e$.
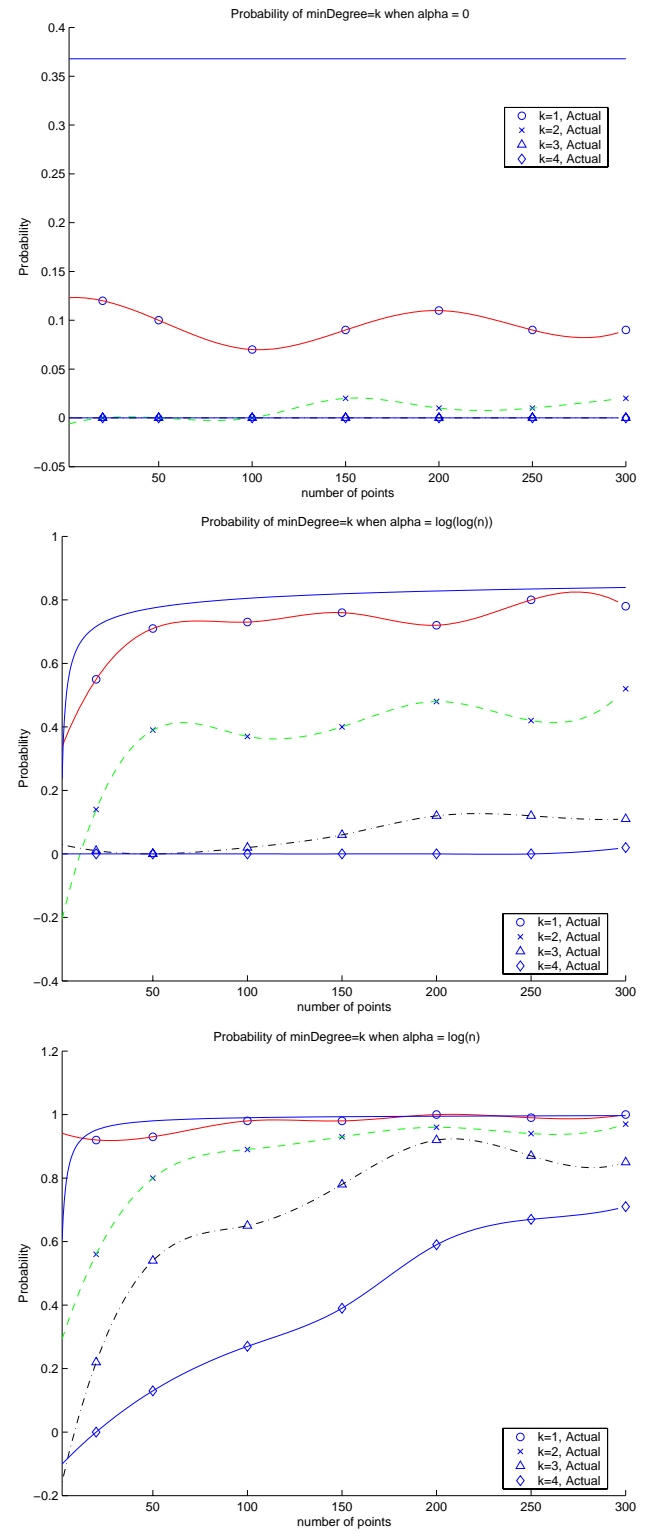

Figure 9: Probability that $G(V, r)$ has min-degree $k$ for theoretical $r$.

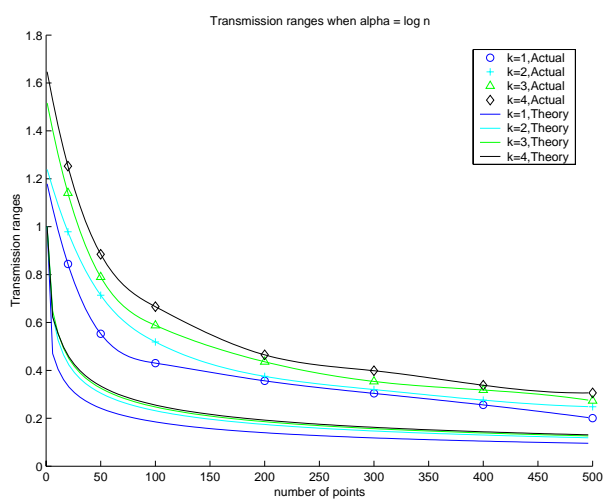

Figure 10: Practical range that $G(V, r)$ is $k$-connected with probability $1-n$. 
$e^{-c}$ and the total number of isolated active nodes is also asymptotically Poisson with mean $p e^{-c}$. It is interesting to consider the $k$-connectivity under the Bernoulli model.

\section{ACKNOWLEDGEMENTS}

The authors would like to thank the anonymous reviewers for valuable comments for improving the paper.

\section{REFERENCES}

[1] M. Bahramgiri, M.T. Hajiaghayi, and V.S. Mirrokni. Fault-tolerant and 3-Dimensional Distributed Topology Control Algorithms in Wireless Multi-hop Networks. In IEEE Int. Conf. on Computer Communications and Networks (ICCCNO2), pages 392-397, 2002.

[2] C. Bettstetter. On the minimum node degree and connectivity of a wireless multihop network. In ACM MobiHoc'02, 2002.

[3] D.M.Blough, M.Leoncini, G.Resta, and P.Santi. On the symmetric range assignment problem in wireless ad hoc networks. In Proc. 2nd IFIP Int. Conf. on Theoretical Computer Science, 2002.

[4] B. Bollobás. Random Graphs. Cambridge University Press, 2001.

[5] P. Bose, P. Morin, I. Stojmenovic, and J. Urrutia. Routing with guaranteed delivery in ad hoc wireless networks. ACM/Kluwer Wireless Networks, 7(6), 2001.

[6] S. Das, C. Perkins, and E. Royer. Performance comparison of two on-demand routing protocols for ad hoc networks. In Proceedings of IEEE INFOCOM, March 2000.

[7] H. Dette and N. Henze. Some peculiar boundary phenomena for extremes of $r$ th nearest neighbor links. Statistics \& Probability Letters, 10:381-390, 1990.

[8] A. Goldberg and S. Rao. Flows in undirected unit capacity networks. Technical Report 97-103, NEC Research Institute, Inc, 1997.

[9] M. Grossglauser and D. Tse. Mobility increases the capacity of ad-hoc wireless networks. In INFOCOMM, vol. 3, pages $1360-1369,2001$.

[10] P. Gupta and P. Kumar. Capacity of wireless networks. Technical report, University of Illinois, Urbana-Champaign, 1999.

[11] P. Gupta and P. R. Kumar. Critical power for asymptotic connectivity in wireless networks. Stochastic Analysis, Control, Optimization and Applications: A Volume in Honor of W.H. Fleming, W. M. McEneaney, G. Yin, and Q. Zhang (Eds.), 1998.

[12] D. B. Johnson and D. A. Maltz. Dynamic source routing in ad hoc wireless networks. In Imielinski and Korth, editors, Mobile Computing, volume 353. Kluwer Academic Publishers, 1996.

[13] L. Li, J. Y. Halpern, P. Bahl, Y.-M. Wang, and R. Wattenhofer. Analysis of a cone-based distributed topology control algorithms for wireless multi-hop networks. In ACM PODC, 2001.

[14] X.-Y. Li, P.-J. Wan, and Y. Wang. Power efficient and sparse spanner for wireless ad hoc networks. In IEEE Int. Conf. on Computer Communications and Networks (ICCCNO1), pages 564-567, 2001.

[15] X.-Y. Li, P.-J. Wan, Y. Wang, and O. Frieder. Sparse power efficient topology for wireless networks. In IEEE Hawaii Int. Conf. on System Sciences (HICSS), 2002.
[16] T. Lukovszki. New Results on Geometric Spanners and Their Applications. PhD thesis, University of Paderborn, 1999.

[17] D. Maltz, J. Broch, J. Jetcheva, and D. Johnson. The effects of on-demand behavior in routing protocols for multi-hop wireless ad hoc networks. IEEE JSAC, August 1999.

[18] S. Narayanaswamy, V. Kawadia, R. Sreenivas, and P. Kumar. Power control in ad-hoc networks: Theory, architecture, algorithm and implementation of the COMPOW protocol. In European Wireless Conference, 2002.

[19] O. D. Patrick. Connectivity in ad-hoc and hybrid networks.

[20] M. Penrose. The longest edge of the random minimal spanning tree. Annals of Applied Probability, 7:340-361, 1997.

[21] M. Penrose. Extremes for the minimal spanning tree on normally distributed points. Advances in Applied Probability, 30:628-639, 1998.

[22] M. Penrose. On k-connectivity for a geometric random graph. Random Structures and Algorithms, 15:145-164, 1999.

[23] M. Penrose. A strong law for the longest edge of the minimal spanning tree. Annals of Probability, 27:246-260, 1999.

[24] C. Perkins. Ad-hoc on-demand distance vector routing. In MILCOM '97, Nov. 1997.

[25] R. Rajaraman. Topology control and routing in ad hoc networks: A survey. In SIGACT News, 33:60-73, 2002.

[26] R. Ramanathan and R. Rosales-Hain. Topology control of multihop wireless networks using transmit power adjustment. In IEEE INFOCOM, 2000.

[27] S. Ramanathan and M. Steenstrup. A survey of routing techniques for mobile communication networks. ACM/Baltzer Mobile Networks and Applications, pages 89-104, 1996.

[28] E. Royer and C. Toh. A review of current routing protocols for ad-hoc mobile wireless networks. IEEE Personal Communications, Apr. 1999.

[29] M. Sanchez, P. Manzoni, and Z. Haas. Determination of critical transmission range in ad-hoc networks. In Multiaccess, Mobility and Teletraffic for Wireless Communications (MMT'99), 1999.

[30] P.Santi and D.M.Blough. An evaluation of connectivity in mobile wireless ad hoc networks. In Proc. IEEE DSN, pages 89-98, 2002.

[31] I. Stojmenovic and X. Lin. Loop-free hybrid single-path/flooding routing algorithms with guaranteed delivery for wireless networks. IEEE Trans. on Parallel and Distributed Systems, 12(10), 2001.

[32] Y. Wang and X.-Y. Li. Distributed spanner with bounded degree for wireless ad hoc networks. In IPDPS: Parallel and Distributed Computing Issues in Wireless networks and Mobile Computing, 2002.

[33] F. Xue and P. R. Kumar. The number of neighbors needed for connectivity of wireless networks.

[34] A. C.-C. Yao. On constructing minimum spanning trees in k-dimensional spaces and related problems. SIAM J. Computing, 11:721-736, 1982.

[35] C.-W. Yi, P.-J. Wan, X.-Y. Li, and O. Frieder. Asymptotic distribution of the number of isolated nodes in wireless ad hoc networks with bernoulli nodes. In Proc. of IEEE WCNC, 2003. 